\title{
Topological transitivity and wandering intervals for group actions on the line $\mathbb{R}$
}

\author{
Enhui Shi \\ School of Mathematical Sciences \\ Soochow University \\ Suzhou 215006, P. R. China \\ E-mail: ehshi@suda.edu.cn \\ Lizhen Zhou \\ School of Mathematical Sciences \\ Soochow University \\ Suzhou 215006, P. R. China \\ E-mail: zhoulizhen@suda.edu.cn
}

\begin{abstract}
For every group $G$, we show that either $G$ has a topologically transitive action on the line $\mathbb{R}$ by orientation-preserving homeomorphisms, or every orientation-preserving action of $G$ on $\mathbb{R}$ has a wandering interval. According to this result, all groups are divided into two types: transitive type and wandering type, and the types of several groups are determined. We also show that every finitely generated orderable group of wandering type is indicable. As a corollary, we show that if a higher rank lattice $\Gamma$ is orderable, then $\Gamma$ is of transitive type.
\end{abstract}

\section{Preliminaries}

Let $X$ be a topological space and let $\operatorname{Homeo}(X)$ be the homeomorphism group of $X$. Suppose $G$ is a group. A group homomorphism $\phi: G \rightarrow$ Homeo $(X)$ is called an action of $G$ on $X$; the action is said to be faithful

2010 Mathematics Subject Classification: Primary 37B05; Secondary 37E99.

Key words and phrases: topological transitivity; orderable group; indicable group. 
if $\phi$ is injective. If $G$ is a subgroup of $\operatorname{Homeo}(X)$, then the action of $G$ on $X$ always refers to the inclusion homomorphism $\iota: G \hookrightarrow \operatorname{Homeo}(X)$. For brevity, we usually use $g x$ or $g(x)$ instead of $\phi(g)(x)$ for $g \in G$ and $x \in X$. The orbit of $x \in X$ under the action of $G$ is the set $G x \equiv\{g x: g \in G\} ; x$ is called a fixed point of $\phi$ or of $G$, if $g x=x$ for all $g \in G$. We use $\operatorname{Fix}(G)$ to denote the fixed point set of $G$; use $\operatorname{Fix}(g)$ to denote the fixed point set of the cyclic group $\langle g\rangle$ generated by $g \in G$. A subset $Y$ of $X$ is called $G$-invariant, if $g(Y) \subset Y$ for all $g \in G$.

Let $\phi$ be an action of group $G$ on a topological space $X$. The action $\phi$ (or $G$ ) is said to be topologically transitive, if for every nonempty open subsets $U$ and $V$ of $X$, there is some $g \in G$ such that $g(U) \cap V \neq \emptyset$. It is well known that, when $G$ is countable and $X$ is a Polish space, $G$ is topologically transitive if and only if there is a point $x \in X$ such that the orbit $G x$ is dense in $X$. Furthermore, $G$ is said to be minimal if for every $x \in X$ the orbit $G x$ is dense in $X$; this is equivalent to saying that there is no proper $G$-invariant nonempty closed subset of $X$. A closed subset $Y$ of $X$ is said to be minimal, if $Y$ is $G$-invariant and the restriction action $\left.G\right|_{Y}$ of $G$ to $Y$ is minimal. An argument using Zorn's lemma shows the existence of minimal sets when $X$ is a compact metric space, but this is not true in general when $X$ is not compact.

Let $\mathbb{R}$ be the real line and let $\mathrm{Homeo}_{+}(\mathbb{R})$ be the orientation-preserving homeomorphism group of $\mathbb{R}$. A group homomorphism $\phi$ from $G$ to $\mathrm{Homeo}_{+}(\mathbb{R})$ is called an orientation-preserving action of $G$ on $\mathbb{R}$. An open interval $(a, b) \subset \mathbb{R}$ is said to be a wandering interval of $\phi$ or of $G$ if, for every $g \in G$, either the restriction $\left.g\right|_{(a, b)}=\operatorname{Id}_{(a, b)}$ or $g((a, b)) \cap(a, b)=\emptyset$.

For any $\alpha \in \mathbb{R}$, define $L_{\alpha}, M_{\alpha}: \mathbb{R} \rightarrow \mathbb{R}$ by letting $L_{\alpha}(x)=x+\alpha$ and $M_{\alpha}(x)=\alpha x$ for every $x \in \mathbb{R}$. We use $\mathbb{Z}^{n}$ to denote the free abelian group of rank $n$. Now we give some examples to illustrate the above notions, which will be used in section 4 .

Example 1.1. Every open interval $(a, b)$ with $b-a<1$ is a wandering interval for the $\mathbb{Z}$ action generated by $L_{1}$.

Example 1.2. Let $\alpha$ be an irrational number, then the $\mathbb{Z}^{2}$ action generated by $L_{1}$ and $L_{\alpha}$ on $\mathbb{R}$ is minimal.

Example 1.3. Let $n$ be a positive integer. Let $T=L_{1}$ and let $S=M_{n}$. Then $S^{-m} T S^{m}(x)=x+n^{-m}$ for all non negative integers $m$ and all $x \in \mathbb{R}$, which clearly implies the minimality of the action of the group $G$ generated by $S$ and $T$. 
Example 1.4. Let $f=L_{1}$ and let $k \geq 2$ be a positive integer. Define a homeomorphism $g$ on $\mathbb{R}$ by setting

$$
g(x)=(x-n)^{2^{(-1)^{n} k^{-n}}}+n
$$

for all integers $n$ and all $x \in[n, n+1)$. Then, for $x \in[n, n+1)$, we have

$$
\begin{aligned}
f g f^{-1}(x) & =(x-1-(n-1))^{2^{(-1)^{n-1} k^{-(n-1)}}}+(n-1)+1 \\
& =(x-n)^{2^{(-1)^{n-1} k^{-(n-1)}}}+n
\end{aligned}
$$

and

$$
g^{-k}(x)=(x-n)^{2^{(-1)^{n+1} k^{-n} k}}+n=(x-n)^{2^{(-1)^{n-1} k^{-(n-1)}}}+n .
$$

So $f g f^{-1}=g^{-k}$. Since $f^{m} g^{l} f^{n}\left(\frac{1}{2}\right)=\left(\frac{1}{2}\right)^{2^{(-1)^{n} k^{-n} l}}+n+m$ for all integers $m, l, n$, the set $\left\{f^{m} g^{l} f^{n}\left(\frac{1}{2}\right): m, l, n \in \mathbb{Z}\right\}$ is dense in $\mathbb{R}$. This implies that the action by the group $\langle f, g\rangle$ generated by $f$ and $g$ is topologically transitive.

\section{Background and main results}

The dynamical system for group actions on one-manifolds has been intensively studied. One may consult [6, 10] for a systematic introduction to this area. Group actions on the real line $\mathbb{R}$ are closely related to the study of orderability of a group. In fact, a countable group $G$ is orderable (that is, $G$ admits a left-invariant total order relation) if and only if it admits a faithful orientation-preserving action on the real line (see [11, Prop. 2.1]). Many important groups coming from geometry and topology are known to be orderable or nonorderable (see e.g. [2, 4, 16, 19]). In addition, an orderable group may possess some interesting algebraic properties (see e.g. [9, 12, 18]).

The purpose of the paper is to classify group actions on $\mathbb{R}$ by means of topological transitivity. One may consult [15] for some related investigations. We first give the following dichotomy theorem.

Theorem 2.1. Let $G$ be a group. Then either $G$ has a topologically transitive action on the line $\mathbb{R}$ by orientation-preserving homeomorphisms, or every orientation-preserving action of $G$ on $\mathbb{R}$ has a wandering interval.

We should note that the "dichotomy phenomenon" in Theorem 2.1 is far from being true for group actions on spaces of dimension $\geq 2$. For example, if $D$ is the closed unit disk in the plane and $S^{2}$ is the unit sphere in $\mathbb{R}^{3}$, then any one point union of $D$ and $S^{2}$ admits no topologically transitive homeomorphism but admits a homeomorphism with no wandering open set.

Theorem 2.1 motivates us to give the following definition. 
Definition 2.2. A group $G$ is of transitive type if it has a topologically transitive action on the line $\mathbb{R}$ by orientation-preserving homeomorphisms; it is of wandering type if every orientation-preserving action of $G$ on $\mathbb{R}$ has a wandering interval.

Recall that a group $G$ is poly-cyclic (resp. super-poly-cyclic) if it admits a decreasing sequence of subgroups $G=N_{0} \triangleright N_{1} \triangleright \ldots \triangleright N_{k}=\{e\}$ for some positive integer $k$ such that $N_{i+1}$ is normal in $N_{i}$ (resp. $N_{i+1}$ is normal in $G$ ) and $N_{i} / N_{i+1}$ is cyclic for each $i \leq k-1$; it is called poly-infinite-cyclic (resp. super-poly-infinite-cyclic) if $N_{i} / N_{i+1}$ is infinitely cyclic for each $i \leq k-1$. It is well known that all poly-cyclic groups are solvable and all finitely generated torsion free nilpotent groups are super-poly-infinite-cyclic.

Suppose $G=N_{0} \triangleright N_{1} \triangleright \ldots \triangleright N_{k}=\{e\}$ is super-poly-infinite-cyclic. Take $f_{i} \in N_{i} \backslash N_{i+1}$ such that $N_{i} / N_{i+1}=\left\langle f_{i} N_{i+1}\right\rangle$ for each $i$. Then $f_{i} f_{i+1} N_{i+2} f_{i}^{-1}=$ $f_{i+1}^{n_{i}} N_{i+2}$ where $n_{i}= \pm 1$. We call the $(k-1)$-tuple $\left(n_{0}, n_{1}, \ldots, n_{k-2}\right)$ the name of $G$. Clearly, the name of $G$ is independent of the choice of $f_{i}$.

For each integer $n$, the Baumslag-Solitar group $B(1, n)$ is the group $\langle a, b$ : $\left.b a=a^{n} b\right\rangle ; B(1,-1)$ is the fundamental group of the Klein Bottle, which is a classical example being of orderable but not bi-orderable (see [10, Exercise 2.2.68]).

Theorem 2.3. The following groups are of transitive type: the nonabelian free group $\mathbb{Z} * \mathbb{Z}$; any super-poly-infinite-cyclic group $G=N_{0} \triangleright N_{1} \triangleright \ldots \triangleright N_{k}=$ $\{e\}$ having the name $\left(n_{0}, n_{1}, \ldots, n_{k-2}\right)$ with some $n_{i}=1$; any poly-infinitecyclic, non super-poly-infinite-cyclic group $G$; the Baumslag-Solitar group $B(1, n)$ with $n \neq 0$ and $n \neq-1$.

The following groups are of wandering type: finite groups; the infinite cyclic group $\mathbb{Z} ; S L(2, \mathbb{Z})$; finite index subgroups of $S L(n, \mathbb{Z})$ with $n \geq 3$; any super-poly-infinite-cyclic group $G=N_{0} \triangleright N_{1} \triangleright \ldots \triangleright N_{k}=\{e\}$ having the name $(-1,-1, \ldots,-1)$; the Baumslag-Solitar group $B(1,-1)$.

Recall that a group is indicable if it has a homomorphism onto the infinite cyclic group. One may consult [1, 7, 18] for the discussions about indicability of orderable groups.

Theorem 2.4. If $G$ is a finitely generated nontrivial orderable group of wandering type, then $G$ is indicable.

$A$ higher rank lattice is a lattice of a simple Lie group with finite center and with real rank $\geq 2$. The 1 -dimensional Zimmer's rigidity conjecture says that every continuous action of a higher rank lattice on the circle $\mathbb{S}^{1}$ must 
factor through a finite group action. Though the conjecture is still open now, Ghys (see [5]) and Burger-Monod (see [3]) proved independently the existence of periodic points for such actions. This implies that 1-dimensional Zimmer's rigidity conjecture is equivalent to that no higher rank lattice is orderable. We get immediately the following corollary by Theorem 2.4 and Theorem 6.5 in the appendix.

Corollary 2.5. Suppose $G$ is a higher rank lattice. If $G$ is orderable, then it is of transitive type.

\section{The dichotomy theorem}

Lemma 3.1. Let $G$ be a group. Suppose $G$ has no topologically transitive action on the line $\mathbb{R}$ by orientation-preserving homeomorphisms. Then, for every action $\phi: G \rightarrow$ Homeo $_{+}(\mathbb{R})$ and for every $x \in \mathbb{R}, \overline{G x}$ is countable; in particular, $\overline{G x}$ is nowhere dense.

Proof. Assume to the contrary that there is some action $\phi_{0}: G \rightarrow$ Homeo $_{+}(\mathbb{R})$ and some $x_{0} \in \mathbb{R}$ such that $\overline{G x_{0}}$ is uncountable. Then, by collapsing the maximal open intervals of $\mathbb{R} \backslash \overline{G x_{0}}$, we get an induced topologically transitive action of $G$ on either $[0,1]$, or $[0,1)$, or $(0,1]$, or $(0,1)$. By removing the endpoints of the phase space of the induced action if necessary, we get a topologically transitive action of $G$ on $\mathbb{R}$ by orientation-preserving homeomorphisms. This contradicts the assumption.

Proof of Theorem 2.1. Assume to the contrary that the following two items hold simultaneously:

(a) $G$ has no topologically transitive action on $\mathbb{R}$ by orientation-preserving homeomorphisms;

(b) there is an action $\phi: G \rightarrow \mathrm{Homeo}_{+}(\mathbb{R})$ such that $\phi$ has no wandering interval.

From Assumption (b) and the definition of wandering interval, there is some $x_{1} \in \mathbb{R}$ and some $g_{1} \in G$ such that $x_{1}<\phi\left(g_{1}\right)\left(x_{1}\right)$ (otherwise, $\phi(g)=\operatorname{Id}_{\mathbb{R}}$ for all $g \in G$; then every open interval in $\mathbb{R}$ is wandering). Without loss of generality, we suppose that $\left\{x_{1}, \phi\left(g_{1}\right)\left(x_{1}\right)\right\} \subset(0,1)$. Set $U_{0}=(0,1)$. For the simplicity of notations, we use $g(x)$ instead of $\phi(g)(x)$ in what follows.

Now we define inductively a sequence of open intervals $U_{i}$ and $g_{i} \in G$, $i=1,2, \ldots$, such that 
(1) for each $i \geq 1, U_{i-1} \supset U_{i}$;

(2) for every $g \in G$ and every $i \geq 1$, either $g\left(U_{i}\right)=U_{i}$, or $g\left(U_{i}\right) \cap U_{i}=\emptyset$;

(3) for every $g \in G$ and every $i \geq 1, \operatorname{diam}\left(g\left(U_{i}\right) \cap[0,1]\right)<\frac{1}{i}$;

(4) for each $i \geq 1, \overline{U_{i}} \cap g_{i}\left(\overline{U_{i}}\right)=\emptyset$ and $\overline{U_{i}} \cup g_{i}\left(\overline{U_{i}}\right) \subset U_{i-1}$.

For $i=1$, take a sufficiently small interval $V_{1} \subset(0,1)$ such that $x_{1} \in V_{1}$,

$$
\overline{V_{1}} \cup g_{1}\left(\overline{V_{1}}\right) \subset U_{0} \text {, and } \overline{V_{1}} \cap g_{1}\left(\overline{V_{1}}\right)=\emptyset \text {. }
$$

Take a sufficiently large positive integer $i_{1}>1$ such that

$$
\frac{1}{i_{1}}<\frac{1}{2} \operatorname{diam}\left(V_{1}\right) \text {. }
$$

Let $A_{1}=\left\{k \frac{1}{i_{1}}: k=0,1, \ldots, i_{1}\right\}$ and let $B_{1}=\overline{G A_{1}}$. It follows from Lemma 3.1 that $B_{1}$ is a nowhere dense $G$-invariant closed subset of $\mathbb{R}$. From (3.2) and the definition of $A_{1}$, there exists a maximal open interval $U_{1}$ of $\mathbb{R} \backslash B_{1}$ such that $U_{1} \subset V_{1}$. Then (1) - (4) hold for $U_{1}$ and $g_{1}$ by (3.1), (3.2) and the definition of $U_{1}$.

Suppose that for $1 \leq i \leq k$ we have defined $U_{i}$ and $g_{i}$ which satisfy $(1)-(4)$. Then define $U_{k+1}$ and $g_{k+1}$ as follows. From Assumption (b), $U_{k}$ is nonwandering, which together with (2) implies that there is some point $x_{k+1} \in U_{k}$ and some $g_{k+1} \in G$ such that $g_{k+1}\left(x_{k+1}\right) \in U_{k}$ and $g_{k+1}\left(x_{k+1}\right)>$ $x_{k+1}$. Take a sufficiently small open interval $V_{k+1}$ such that $x_{k+1} \in V_{k+1}$,

$$
\overline{V_{k+1}} \cup g_{k+1}\left(\overline{V_{k+1}}\right) \subset U_{k} \text {, and } \overline{V_{k+1}} \cap g_{k+1}\left(\overline{V_{k+1}}\right)=\emptyset \text {. }
$$

Take a sufficiently large positive integer $i_{k+1}>k+1$ such that

$$
\frac{1}{i_{k+1}}<\frac{1}{2} \operatorname{diam}\left(V_{k+1}\right)
$$

Let $A_{k+1}=\left\{k \frac{1}{i_{k+1}}: k=0,1, \ldots, i_{k+1}\right\}$ and let $B_{k+1}=\overline{G A_{k+1}}$. Similar to the case of $i=1$, we get a maximal open interval $U_{k+1}$ of $\mathbb{R} \backslash B_{k+1}$ which satisfy the conditions (1) - (4).

Now we define a sequence of subsets $G_{i}$ of $G$ for $i=1,2, \ldots$ as follows. Let $G_{1}=\left\{e, g_{1}\right\}$. Assume $G_{i}$ have been defined for $1 \leq i \leq k$. Then let $G_{k+1}=G_{k} \cup\left\{g g_{k+1}: g \in G_{k}\right\}$. For each $k=1,2, \ldots$, set $\Lambda_{k}=\cup_{g \in G_{k}} g\left(\overline{U_{k}}\right)$, and set $\Lambda=\cap_{k=1}^{\infty} \Lambda_{k}$. It follows from (3) and (4) that $\Lambda$ is homeomorphic to the Cantor set, and, for any point $x \in \Lambda, \overline{G x} \supset \Lambda$ (one may see Fig. 1 for the illustration of the ideas of the construction). This implies that $G$ has a topologically transitive orientation-preserving action on $\mathbb{R}$ by Lemma 3.1, which contradicts the assumption (a). 


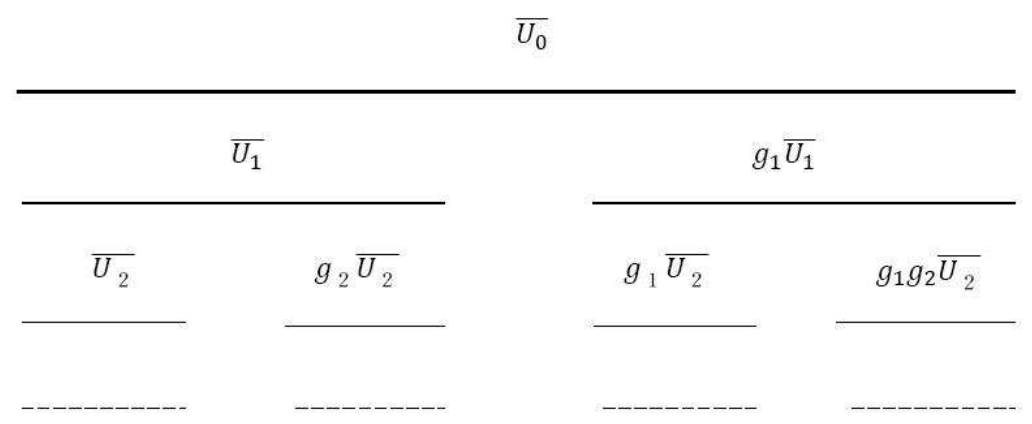

Fig. 1

\section{Types of some groups}

In this section, we start to prove Theorem 2.3. From Definition 2.2, we immediately have that all finite groups and the infinite cyclic group $\mathbb{Z}$ are of wandering type.

Proposition 4.1. Suppose $G=N_{0} \triangleright N_{1} \triangleright \ldots \triangleright N_{k}=\{e\}$ is super-polyinfinite-cyclic and has the name $(-1,-1, \ldots,-1)$. Then $G$ is of wandering type.

Proof. By the hypothesis, we can take a sequence $g_{i} \in N_{i} \backslash N_{i+1}$ and take $g_{k}=e$ such that $N_{i}=\left\langle g_{i}, \ldots, g_{k-1}\right\rangle$ and

$$
g_{i} g_{i+1} N_{i+2}=g_{i+1}^{-1} g_{i} N_{i+2} \text { for each } i: 0 \leq i \leq k-2 \text {. }
$$

Let $\phi: G \rightarrow$ Homeo $_{+}(\mathbb{R})$ be any orientation-preserving action of $G$ on $\mathbb{R}$ and let $f_{i}=\phi\left(g_{i}\right)$ for each $i$. If $f_{i}=\operatorname{Id}_{\mathbb{R}}$ for each $i$, then every open interval in $\mathbb{R}$ is wandering by Definition 2.2 . So, we may as well suppose that

$$
\mathbb{R} \backslash \operatorname{Fix}\left(N_{k-1}\right) \neq \emptyset \text {. }
$$

Claim A. There exists a sequence of open intervals $J_{i}, 0<i \leq k$, such that $J_{1} \supset \ldots \supset J_{k}, J_{i} \subset \mathbb{R} \backslash \operatorname{Fix}\left(f_{i}\right)$ for $i<k, f_{i-1}\left(J_{i}\right) \cap J_{i}=\emptyset$ and $f_{j}\left(J_{i}\right)=J_{i}$ for $j \geq i$.

We prove this claim by induction. Take a maximal open interval $J_{k-1}$ in $\mathbb{R} \backslash \operatorname{Fix}\left(N_{k-1}\right)$ by (4.2), and take an open interval $J_{k} \subset J_{k-1}$ such that $f_{k-1}\left(J_{k}\right) \cap J_{k}=\emptyset$. Assume that, for some $l \geq 0$, we have obtained open intervals $J_{l+1} \supset \ldots \supset J_{k}$ such that $J_{i} \subset \mathbb{R} \backslash \operatorname{Fix}\left(f_{i}\right)$ for each $i: k>i>l$, $f_{i-1}\left(J_{i}\right) \cap J_{i}=\emptyset$ for $i>l$, and $f_{j}\left(J_{i}\right)=J_{i}$ for $j \geq i$. Let $J_{i}=\left(\alpha_{i}, \beta_{i}\right), k \geq$ $i \geq l+1$. Let $J_{l}=\left(\alpha_{l}, \beta_{l}\right)$ be the maximal open interval of $\mathbb{R} \backslash \operatorname{Fix}\left(f_{l}\right)$, which contains $J_{l+1}$. Since either $\alpha_{l}=\lim _{m \rightarrow+\infty} f^{m}\left(\alpha_{l+1}\right)$ or $\alpha_{l}=\lim _{m \rightarrow-\infty} f^{m}\left(\alpha_{l+1}\right)$, and 
$\alpha_{l+1} \in \operatorname{Fix}\left(N_{l+1}\right)$, we have $\alpha_{l} \in \operatorname{Fix}\left(N_{l}\right)$. Similarly, $\beta_{l} \in \operatorname{Fix}\left(N_{l}\right)$. So, $f_{j}\left(J_{l}\right)=$ $J_{l}$ for $j \geq l$. In addition, we have $f_{l-1}\left(J_{l}\right) \cap J_{l}=\emptyset$. Otherwise, $f_{l-1}\left(J_{l}\right)=$ $J_{l}$ since $f_{l-1}\left(\operatorname{Fix}\left(N_{l}\right)\right)=\operatorname{Fix}\left(N_{l}\right)$ by (4.1). Without loss of generality, we suppose $f_{l}(x)>x$ for every $x \in J_{l}$. Let $w=\alpha_{l+1}$. Then $w \in \operatorname{Fix}\left(N_{l+1}\right)$, and $\alpha_{l}=\lim _{m \rightarrow+\infty} f_{l-1} f_{l}^{-m}(w)=\lim _{m \rightarrow+\infty} f_{l}^{m}\left(f_{l-1}(w)\right)=\beta_{l}$ by (4.1), which is a contradiction. Thus we complete the proof of Claim A.

Claim B. $J_{k}$ is a wandering interval of $\phi$. In fact, for any $g \in G, \phi(g)$ can be expressed as $\phi(g)=f_{0}^{n_{0}} f_{1}^{n_{1}} \ldots f_{k-1}^{n_{k-1}}$ for some integers $n_{0}, n_{1}, \ldots, n_{k-1}$. If $\phi(g)\left(J_{k}\right) \cap J_{k} \neq \emptyset$, then it follows from Claim A that $n_{0}=n_{1}=\ldots=$ $n_{k-1}=0$. Thus $\phi(g)=\operatorname{Id}_{\mathbb{R}}$.

From Claim B, we get that $G$ is of wandering type.

Lemma 4.2. Let $H$ be a normal subgroup of $G$ such that $G / H$ is infinite cyclic. Then $G$ is of transitive type provided that $H$ is of transitive type.

Proof. Suppose $G / H=\langle a H\rangle$ for some $a \in G \backslash H$. Let $a$ act on the line by the unit translation $\phi(a): x \mapsto x+1$. By the assumption, $H$ has an orientation-preserving topologically transitive action on $(0,1)$, which extends to an action $\phi$ on the interval $[0,1]$ by fixing the endpoints. Then extend this $H$ action to $G$ action on the line by setting, for each $j \in \mathbb{Z}$,

$$
\phi(b)(x)=\phi\left(a^{-j} b a^{j}\right)(x-j)+j
$$

for all $x \in[j, j+1]$ and $b \in H$. Then define $\phi$ on $G$ by setting $\phi\left(a^{i} b\right)=$ $(\phi(a))^{i} \phi(b)$ for all $i \in \mathbb{Z}$ and $b \in H$. It is direct to check that $\phi\left(g_{1} g_{2}\right)=$ $\phi\left(g_{1}\right) \phi\left(g_{2}\right)$ for arbitrary two elements $g_{1}=a^{l_{1}} b_{1}$ and $g_{2}=a^{l_{2}} b_{2}$ in $G$ where $b_{1}, b_{2} \in H$. In fact, for all $x \in[i, i+1]$, we have

$$
\begin{aligned}
\phi\left(g_{1} g_{2}\right)(x) & =\phi\left(a^{l_{1}} b_{1} a^{l_{2}} b_{2}\right)(x) \\
& =\phi\left(a^{l_{1}+l_{2}} a^{-l_{2}} b_{1} a^{l_{2}} b_{2}\right)(x) \\
& =\phi\left(a^{l_{1}+l_{2}}\right) \phi\left(a^{-l_{2}} b_{1} a^{l_{2}} b_{2}\right)(x) \\
& =\phi\left(a^{-i} a^{-l_{2}} b_{1} a^{l_{2}} b_{2} a^{i}\right)(x-i)+i+l_{1}+l_{2}
\end{aligned}
$$

while

$$
\begin{aligned}
\phi\left(g_{1}\right) \phi\left(g_{2}\right)(x) & =\phi\left(a^{l_{1}} b_{1}\right) \phi\left(a^{l_{2}} b_{2}\right)(x) \\
& =\phi\left(a^{l_{1}} b_{1}\right)\left(\phi\left(a^{-i} b_{2} a^{i}\right)(x-i)+i+l_{2}\right) \\
& =\phi\left(a^{-i-l_{2}} b_{1} a^{i+l_{2}}\right) \phi\left(a^{-i} b_{2} a^{i}\right)(x-i)+i+l_{1}+l_{2} \\
& =\phi\left(a^{-i} a^{-l_{2}} b_{1} a^{l_{2}} b_{2} a^{i}\right)(x-i)+i+l_{1}+l_{2}
\end{aligned}
$$

as required. Thus $\phi$ is an orientation-preserving action of $G$ on the line and the topological transitivity of $\phi$ is clear. 
Proposition 4.3. Suppose $G=N_{0} \triangleright N_{1} \triangleright \ldots \triangleright N_{k}=\{e\}$ is super-polyinfinite-cyclic with $k \geq 2$ and has the name $\left(n_{0}, n_{1}, \ldots, n_{k-2}\right)$ with some $n_{i}=1$. Then $G$ is of transitive type.

Proof. If $n_{k-2}=1$, then $N_{k-2}$ is isomorphic to $\mathbb{Z}^{2}$, which is of transitive type by Example 1.2. By repeated applications of Lemma 4.2, we get that $G$ is of transitive type. If $n_{i}=1$ for some $i<k-2$, then $G / N_{i+2}$ is of transitive type by the previous argument. Since $G / N_{i+2}$ is a factor of $G, G$ is of transitive type.

Proposition 4.4. Suppose $G$ is poly-infinite-cyclic. If $G$ is not super-polycyclic, then $G$ is of transitive type.

Proof. By Proposition 6.4 in the appendix, there is a decreasing sequence of normal subgroups of $G$ : $G=N_{0} \triangleright N_{1} \triangleright \ldots \triangleright N_{k}=\{e\}$ for some $k>0$, such that each $N_{i} / N_{i+1}$ is isomorphic to $\mathbb{Z}^{d_{i}}$ for some $d_{i} \geq 1$. If every $N_{i} / N_{i+1}$ is isomorphic to $\mathbb{Z}$, then $G$ is super-poly-cyclic, which contradicts the assumption. So, there is some $i^{\prime}$ such that $N_{i^{\prime}} / N_{i^{\prime}+1}$ is isomorphic to $\mathbb{Z}^{d_{i^{\prime}}}$ with $d_{i^{\prime}} \geq 2$. Let $\tilde{G}=G / N_{i^{\prime}+1}$. Since $\tilde{N}_{i^{\prime}} \equiv N_{i^{\prime}} / N_{i^{\prime}+1}$ is of transitive type by Example 1.2, $\tilde{G}$ is of transitive type by repeated applications of Lemma 4.2 (note that $\tilde{G} / \tilde{N}_{i^{\prime}}$ is poly-infinite-cyclic). Then $G$ is of transitive type since $\tilde{G}$ is a factor of $G$.

Proposition 4.5. The Baumslag-Solitar group $B(1, n)$ is of transitive type if and only if $n \neq 0$ and $n \neq-1$.

Proof. $(\Rightarrow)$ Suppose $B(1, n)=\left\langle a, b: b a=a^{n} b\right\rangle$. If $n=0$, then $B(1, n)$ is an infinite cyclic group; in this case, every orientation-preserving action of $B(1, n)$ on $\mathbb{R}$ has a wandering interval. Supppose $n=-1$. Let $\phi$ : $B(1,-1) \rightarrow$ Homeo $_{+}(\mathbb{R})$ be any orientation-preserving action of $B(1,-1)$ on $\mathbb{R}$. Let $g=\phi(a)$ and $f=\phi(b)$; then $f g=g^{-1} f$. We wish to show that $\phi$ has a wandering interval. If $\operatorname{Fix}(g)=\mathbb{R}$, then $\phi$ factors through a cyclic group action on $\mathbb{R}$, which ensures the existence of wandering intervals. So, we may suppose that $\operatorname{Fix}(g) \neq \mathbb{R}$.

Claim A. $f(\operatorname{Fix}(g))=\operatorname{Fix}(g)$. In fact, let $x \in \operatorname{Fix}(g)$. Then $g^{-1} f(x)=$ $f g(x)=f(x)$. So, $f(x) \in \operatorname{Fix}\left(g^{-1}\right)=\operatorname{Fix}(g)$. On the other hand, $g^{-1} f^{-1}(x)=$ $f^{-1} g(x)=f^{-1}(x)$, which means $f^{-1}(x) \in \operatorname{Fix}\left(g^{-1}\right)=\operatorname{Fix}(g)$.

From Claim A, we see that $f$ permutes the maximal open intervals in $\mathbb{R} \backslash \operatorname{Fix}(g)$. Fix a maximal open interval $(u, v)$ in $\mathbb{R} \backslash \operatorname{Fix}(g)$ ( $u$ may be $-\infty$, and $v$ may be $+\infty)$. 
Claim B. $f((u, v)) \cap(u, v)=\emptyset$. Otherwise, $f((u, v))=(u, v)$. Without loss of generality, we suppose $g(x)>x$ for every $x \in(u, v)$. Fix any point $w \in(u, v)$, then $v=\lim _{i \rightarrow+\infty} f g^{i}(w)=\lim _{i \rightarrow+\infty} g^{-i}(f(w))=u$ by the relation $f g=g^{-1} f$. This is a contradiction.

From Claim A and Claim B, we immediately get

Claim C. Suppose $f^{m_{1}} g^{n_{1}} f^{m_{2}} g^{n_{2}} \ldots f^{m_{l}} g^{m_{l}}((u, v))=(u, v)$ for some integers $l, m_{i}$, and $n_{i}(1 \leq i \leq l)$. Then $m_{1}+m_{2}+\ldots+m_{l}=0$.

Take an open interval $J \subset(u, v)$ such that $J \cap g(J)=\emptyset$.

Claim D. If $h(J) \cap J \neq \emptyset$ for some $h \in \phi(B(1,-1))$, then $h=\operatorname{Id}_{\mathbb{R}}$. In fact, suppose $h=f^{m_{1}} g^{n_{1}} f^{m_{2}} g^{n_{2}} \ldots f^{m_{l}} g^{m_{l}}$ such that $\left|m_{1}\right|+\left|n_{1}\right|+\left|m_{2}\right|+$ $\left|n_{2}\right|+\ldots+\left|m_{l}\right|+\left|n_{l}\right|$ attains minimum among all expressions of $h$ by $f$ and $g$. This implies that all $m_{i}$ with $m_{i} \neq 0$ have the same signs by the relation $f^{-1} g f=g^{-1}$. However, this forces all $m_{i}=0$ by Claim $\mathrm{C}$ (noting that $h((u, v))=(u, v))$. Then $h=\operatorname{Id}_{\mathbb{R}}$, since $J \cap g(J)=\emptyset$.

It follows from Claim $\mathrm{D}$ that $J$ is a wandering interval for $\phi$.

$(\Leftarrow)$ Since $B(1,1)$ is isomorphic to $\mathbb{Z}^{2}$, it is of transitive type by Example 1.2. If $n>1$, then $B(1, n)$ is of transitive type by Example 1.3 (note that $T$ and $S$ in Example 1.3 satisfy the relation $S T=T^{n} S$ ). In Example 1.4, we see that $f$ and $g$ satisfy the relation $f g=g^{-k} f$ with $k \geq 2$, which implies that $B(1, n)$ is of transitive type when $n \leq-2$.

From example 1.1 , we see that the free nonabelian group $\mathbb{Z} * \mathbb{Z}$ has a topologically transitive action on $\mathbb{R}$ by orientation-preserving homeomorphisms, since $\mathbb{Z}^{2}$ is a factor of $\mathbb{Z} * \mathbb{Z}$. In fact, we can further require the action to be faithful as the following lemma shows.

Proposition 4.6. The free nonabelian group $\mathbb{Z} * \mathbb{Z}$ has a faithful topologically transitive action on $\mathbb{R}$ by orientation-preserving homeomorphisms.

Proof. Let $f, g \in$ Homeo $_{+}(\mathbb{R})$ be defined by $f(x)=x+1$ and $g(x)=x^{3}$ for every $x \in \mathbb{R}$. Then for any nonempty open intervals $U$ and $V$ in $\mathbb{R}$, we have $\operatorname{diam}\left(g^{n}(U)\right)>1$ for some integer $n$, and then there is some integer $m$ such that $f^{m}\left(g^{n}(U)\right) \cap V \neq \emptyset$. Thus the action by the group $H$ generated by $f$ and $g$ is topologically transitive. By the main result in [17], we see that $H$ is isomorphic to $\mathbb{Z} * \mathbb{Z}$.

The following theorem is due to D. Witte-Morris (see [19]).

Theorem 4.7 (Witte-Morris). The group $S L(2, \mathbb{Z})$ and all finite index subgroups of $S L(n, \mathbb{Z})$ with $n \geq 3$ are non-orderable. 
Proposition 4.8. The group $S L(2, \mathbb{Z})$ and all finite index subgroups of $S L(n, \mathbb{Z})$ with $n \geq 3$ are of wandering type.

Proof. Since $S L(2, \mathbb{Z})$ is generated by elements with finite orders, any orientation preserving action of $S L(2, \mathbb{Z})$ on $\mathbb{R}$ must be trivial. So, $S L(2, \mathbb{Z})$ is of wandering type. Suppose $n \geq 3$ and $H$ is a subgroup of $S L(n, \mathbb{Z})$ with finite index. Assume $H$ is of transitive type and let $\phi: H \rightarrow \mathrm{Homeo}_{+}(\mathbb{R})$ be a topologically transitive action of $H$ on $\mathbb{R}$. By Selberg's Lemma (see Theorem 6.6 in the appendix), there is a torsion free normal subgroup $F$ of $H$ which has finite index in $H$. It follows from Theorem 6.5 (see the appendix) and the topological transitivity of $\phi$ that $\operatorname{Ker}(\phi)$ is finite, which implies that $\operatorname{Ker}(\phi) \cap F$ is trivial. So the restriction $\left.\phi\right|_{F}: F \rightarrow \mathrm{Homeo}_{+}(\mathbb{R})$ is injective. Thus $F$ is orderable, which contradicts Theorem 4.7.

Remark 4.9. Since the free non-abelian group $\mathbb{Z} * \mathbb{Z}$ is a finite index subgroup of $S L(2, \mathbb{Z})$ and $\mathbb{Z} * \mathbb{Z}$ is of transitive type, Proposition 4.8 does not hold for finite index subgroups of $S L(2, \mathbb{Z})$.

Then Theorem 2.3 follows from all the propositions in this section.

\section{Indicability}

To prove Theorem 2.4, we need several well-known results about group actions on $\mathbb{R}$. The following lemma can be shown by the dynamical realization method (see [10, Theorem 2.2.19] and its remark).

Lemma 5.1. Every countable nontrivial orderable group has a faithful orientationpreserving action on the line $\mathbb{R}$ without fixed points.

The following lemma is the combination of [10, Proposition 2.1.12] and the remarks after it (see also [8]).

Lemma 5.2. If $G$ is a finitely generated group acting on the line $\mathbb{R}$ by orientation-preserving homeomorphisms, then $G$ admits a nonempty minimal closed subset $\Lambda$ of $\mathbb{R}$, and $\Lambda$ has four possibilities:

(a) $\Lambda$ is a point (in this case, $\Lambda$ is a fixed point of $G$ );

(b) $\Lambda$ is an infinite sequence $\left(a_{n}\right)_{n \in \mathbb{Z}}$ satisfying $a_{n}<a_{n+1}$ for all $n$ and without accumulation points in $\mathbb{R}$;

(c) $\Lambda$ is locally a Cantor set;

(d) $\Lambda=\mathbb{R}$. 
Proof of Proposition 2.5. Suppose $G$ is a finitely generated nontrivial orderable group of wandering type. From Lemma 5.1, we can fix a faithful action $\phi: G \rightarrow \mathrm{Homeo}_{+}(\mathbb{R})$ without fixed points. By Lemma 5.2, there is a minimal set $\Lambda \subset \mathbb{R}$. Since $\phi$ has no fixed points, $\Lambda$ cannot be a single point. By Definition 2.2 and Lemma 3.1, we see that $\Lambda$ is countable, which together with Lemma 5.2 implies that $\Lambda$ is an infinite sequence $\left(a_{n}\right)_{n \in \mathbb{Z}}$ satisfying $a_{n}<a_{n+1}$ for all $n$ and without accumulation points in $\mathbb{R}$. Set $H=\left\{g \in G: g\left(\left(a_{0}, a_{1}\right)\right)=\left(a_{0}, a_{1}\right)\right\}$ and fix an $f \in G$ with $f\left(a_{0}\right)=a_{1}$. By the structure of $\Lambda$, we have $H=\left\{g \in G: g\left(a_{n}\right)=a_{n}\right.$ for all $\left.n\right\}$ and $f\left(a_{n}\right)=a_{n+1}$ for all $n$. Thus $H$ is normal in $G$, and $G / H=\left\{f^{n} H, n \in \mathbb{Z}\right\}$ which is an infinite cyclic group. This completes the proof.

\section{Appendix}

In this section, we first supply some basic results about poly-cyclic groups, which have been used in the precious sections. One may consult [13] for more details.

Proposition 6.1. Let $G$ be a poly-cyclic group and let $H$ be a subgroup of $G$. Then $H$ is poly-cyclic.

Proposition 6.2 ([12]). Let $G$ be a poly-cyclic group. Then $G$ is polyinfinite-cyclic if and only if $G$ is orderable.

Since every subgroup of an orderable group is orderable, we immediately get the the following corollary by Proposition 6.1 and Proposition 6.2.

Corollary 6.3. Let $G$ be a poly-infinite-cyclic group. Then every non-trivial subgroup of $G$ is poly-infinite-cyclic.

Suppose $G$ is a poly-cyclic group. Then $G=N_{0} \triangleright N_{1} \triangleright \ldots \triangleright N_{k}=\{e\}$ with $N_{i} / N_{i+1}$ cyclic for each $i$. The cyclic groups $N_{i} / N_{i+1}$ are called the cyclic factors. The Hirsch number of $G$ is the number of infinite cyclic factors among these $N_{i} / N_{i+1}$. It is an invariant of polycyclic groups.

Proposition 6.4. Let $G$ be a poly-infinite-cyclic group. Then there is a decreasing sequence of normal subgroups of $G$ : $G=G_{0} \triangleright G_{1} \triangleright \ldots \triangleright G_{l}=\{e\}$ for some $l>0$, such that $G_{i} / G_{i+1}$ is a free abelian group of finite rank for each $i$.

Proof. Suppose $G=N_{0} \triangleright N_{1} \triangleright \ldots \triangleright N_{k}=\{e\}$ for some $k>0$, where $N_{i+1}$ is normal in $N_{i}$ and $N_{i} / N_{i+1}$ is infinitely cyclic for each $i \geq 0$. Since $G / N_{1}$ 
is abelian, the commutator group $[G, G] \subset N_{1}$. So, $G /[G, G]$ is isomorphic to $\mathbb{Z}^{d} \times F$ for some $d>1$, where $F$ is a finite abelian group. Let $\pi: G \rightarrow$ $G /[G, G]$ be the quotient homomorphism and let $G_{1}=\pi^{-1}(F)$. Then $G_{1}$ is a character subgroup of $G$ and $G / G_{1}$ is isomorphic to $\mathbb{Z}^{d}$. Similarly, we can get a character subgroup $G_{2}$ of $G_{1}$ (which is also a character subgroup of $G$ ) such that $G_{1} / G_{2}$ is a free abelian group of finite rank, since $G_{1}$ is still poly-infinite-cyclic by Proposition 6.3. Going on in this way, we get a sequence of character subgroups of $G: G=G_{0} \triangleright G_{1} \triangleright \ldots \triangleright G_{i} \triangleright \ldots$ such that $G_{i} / G_{i+1}$ is a free abelian group of finite rank for each $i$. Since the Hirsch number of $G$ is finite, there exists a positive integer $l$ such that $G_{l}=\{e\}$. Thus we complete the proof.

The following theorem is due to Margulis and Kazhdan (see e.g. [20, Theorem 8.1.2]).

Theorem 6.5. Let $\Gamma$ be a higher rank lattice and let $H$ be a normal subgroup of $\Gamma$. Then either $H$ is finite or $\Gamma / H$ is finite.

The following theorem is known as Selberg's Lemma (see [14]).

Theorem 6.6. Let $G$ be a finitely generated subgroup of $G L(n, \mathbb{C})$. Then $G$ contains a torsion free normal subgroup $H$ with finite index in $G$.

\section{Acknowledgements}

The work is supported by NSFC (No. 11771318, No. 11790274). We would like to thank Prof. Binyong Sun for providing us the idea of the proof of Proposition 6.4.

\section{References}

[1] G. Bergman, Right-orderable groups that are not locally indicable, Pac. J. Math. 147 (1991), 243-248.

[2] S. Boyer, D. Rolfsen, B. Wiest, Orderable 3-manifold groups, Ann. Inst. Fourier (Grenoble) 55 (2005), 243-288.

[3] M. Burger, N. Monod, Bounded cohomology of lattices in higher rank Lie groups, J. Eur. Math. Soc. 1 (1999), 199-235.

[4] P. Dehornoy, I. Dynnikov, D. Rolfsen, B. Wiest, Why are braids orderable?, Panoramas et Synthses 14, Socit Mathmatique de France, Paris, 2002. 
[5] É. Ghys, Actions de réseaux sur le cercle, Invent. Math. 137 (1999), 199-231.

[6] É. Ghys, Groups acting on the circle, L'Enseign. Math. 47 (2001), 329-407.

[7] P. Longobardi, M. Maj, A. Rhemtulla, When is a right orderable group locally indicable?, Proc. Amer. Math. Soc. 128 (2000), 637-641.

[8] A. Malyutin, Classification of group actions on the line and the circle. (Russian) Algebra i Analiz 19 (2007), no. 2, 156-182; translation in St. Petersburg Math. J. 19 (2007), 279-296.

[9] A. Navas, Groups, orders, and laws, Groups Geom. Dyn. 8 (2014), 863-882.

[10] A. Navas, Groups of circle diffeomorphisms, Translation of the 2007 Spanish edition, Chicago Lectures in Mathematics, University of Chicago Press, Chicago, IL, 2011.

[11] A. Navas, On the dynamics of (left) orderable groups, Ann. Inst. Fourier (Grenoble) 60 (2010), 1685-1740.

[12] A. Rhemtulla, Polycyclic right-ordered groups, in: Algebra (Carbondale, 1980), Lecture Notes in Math. 848, Springer, Berlin, 1981, 230234.

[13] D. Segal, Polycyclic groups, Cambridge University Press, 1983.

[14] A. Selberg, On discontinuous groups in higher-dimensional spaces, Contributions to Function Theory, Tata Institute, Bombay, 1960.

[15] E. Shi, L. Zhou, Topological conjugation classes of tightly transitive subgroups of $\mathrm{Homeo}_{+}(\mathbb{R})$, Colloq. Math. 145 (2016), 111-120.

[16] H. Short, B. Wiest, Orderings of mapping class groups after Thurston, Enseign. Math. (2) 46 (2000), 279-312.

[17] S. White, The group generated by $x \rightarrow x+1$ and $x \rightarrow x^{p}$ is free, J. Algebra 118 (1988), 408-422.

[18] D. Witte-Morris, Amenable groups that act on the line, Algebr. Geom. Topol. 6 (2006), 2509-2518. 
[19] D. Witte-Morris, Arithmetic groups of higher Q-rank cannot act on 1-manifolds, Proc. Amer. Math. Soc. 122 (1994), 333-340.

[20] R. Zimmer, Ergodic theory and semisimple groups, MMA 81, Birkhäuser, 1984. 